BMJ Open

Diabetes

Research

\& Care

\title{
Risk of diabetes associated with fatty acids in the de novo lipogenesis pathway is independent of insulin sensitivity and response: the Insulin Resistance Atherosclerosis Study (IRAS)
}

\author{
Waqas Qureshi, ${ }^{1}$ Ingrid D Santaren, ${ }^{2}$ Anthony J Hanley, ${ }^{\circ}$ Steven M Watkins, ${ }^{3}$ \\ Carlos Lorenzo, ${ }^{4}$ Lynne E Wagenknecht ${ }^{5}$
}

To cite: Qureshi W, Santaren ID, Hanley AJ, et al. Risk of diabetes associated with fatty acids in the de novo lipogenesis pathway is independent of insulin sensitivity and response: the Insulin Resistance Atherosclerosis Study (IRAS). BMJ Open Diab Res Care 2019;7:e000691. doi:10.1136/ bmjdrc-2019-000691

WQ and IDS contributed equally.

Received 20 April 2019 Revised 11 August 2019 Accepted 19 August 2019
Check for updates

\section{(C) Author(s) (or their} employer(s)) 2019. Re-use permitted under CC BY-NC. No commercial re-use. See rights and permissions. Published by BMJ.

For numbered affiliations see end of article.

Correspondence to Dr Anthony J Hanley; anthony.hanley@utoronto.ca

\section{ABSTRACT}

Objective To examine the associations of fatty acids in the de novo lipogenesis (DNL) pathway, specifically myristic acid (14:0), palmitic acid (16:0), cis-palmitoleic acid (c16:1 n-7), cis-myristoleic acid (c14:1n5), stearic acid (18:0) and cis-oleic acid (c18:1 n-9), with 5-year risk of type 2 diabetes. We hypothesized that DNL fatty acids are associated with risk of type 2 diabetes independent of insulin sensitivity.

Research design and methods We evaluated 719 (mean age $55.1 \pm 8.5$ years, $44.2 \%$ men, $42.3 \%$ Caucasians) participants from the Insulin Resistance Atherosclerosis Study. Multivariable logistic regression models with and without adjustment of insulin sensitivity were used to assess prospective associations of DNL fatty acids with incident type 2 diabetes.

Results Type 2 diabetes incidence was $20.3 \%$ over 5 years. In multivariable regression models, palmitic, palmitoleic, myristic, myristoleic and oleic acids were associated with increased risk of type 2 diabetes $(\mathrm{p}<0.05)$. Palmitic acid had the strongest association (OR per standard unit of palmitic acid $1.46 ; 95 \% \mathrm{Cl} 1.23$ to $1.76 ; p<0.001)$, which remained similar with addition of insulin sensitivity and acute insulin response (AIR) to the model (OR 1.36; 95\% Cl 1.09 to 1.70, $p=0.01$ ). Oleic and palmitoleic acids were also independently associated with incident type 2 diabetes. In multivariable models, ratios of fatty acids corresponding to stearoyl $\mathrm{COA}$ desaturase- 1 and Elovl6 enzymatic activity were significantly associated with risk of type 2 diabetes independent of insulin sensitivity and AIR.

Conclusions We observed associations of DNL fatty acids with type 2 diabetes incidence independent of insulin sensitivity.

\section{INTRODUCTION}

Worldwide, the number of individuals suffering from diabetes mellitus (DM) is projected to increase by $48 \%$ from 451 million adults in 2017 to 693 million by $2045 .{ }^{1}$ In the USA alone, diabetes prevalence was reported to be $7.6 \%$ in 2017 , contributing to an economic burden of $\$ 327$ billion, due

\section{Significance of this study}

What is already known about this subject?

- Fatty acids involved in de novo fatty acid synthesis are known to be associated with risk of diabetes.

What are the new findings?

- The association of fatty acids with risk of diabetes is independent of insulin resistance and pancreatic insulin secretion response. The pathways involving stearoyl CoA desaturase and Elovl6 enzymes might be key factors in this association.

How might these results change the focus of research or clinical practice?

- This research suggests alternative pathways via which fatty acids may increase risk of diabetes other than insulin sensitivity and insulin secretion such as de novo hepatic lipogenesis pathway. We observed that the higher palmitic acid to palmitoleic acid ratio and oleic to stearic acid ratio were associated with higher risk of diabetes. Inhibition of these pathways may provide a new therapeutic method to prevent diabetes. Effective and non-toxic inhibitors may be developed that could prevent from development of diabetes in the future.

to direct medical costs and reduced productivity; this figure has increased by $26 \%$ from 2012 estimates. $^{2}$

Modulation of insulin resistance remains the most common therapeutic pathway amenable to prevention of type 2 diabetes. However, growing evidence suggests that there are several other pathways that could be responsible for development of type 2 diabetes. Fatty acids involved in de novo lipogenesis (DNL) pathway have recently been characterized as a critical pathway in the pathogenesis of type 2 diabetes. Several studies have examined individual fatty acids involved in DNL as possible 
risk factors of type 2 diabetes. ${ }^{3-8}$ However, the results have been inconsistent and a pathophysiological approach to examine the surrogates of enzymes regulating these pathways has been lacking. Moreover, these studies were limited in ethnic diversity.

Therefore, we examined the associations of fatty acids in the DNL pathway, including myristic acid (14:0), palmitic acid (16:0), cis-palmitoleic acid (c16:1 n-7), cis-myristoleic acid (c14:1n5), stearic acid (18:0) and cisoleic acid (c18:1 n-9), with and without adjustment of insulin sensitivity and acute insulin response (AIR), in a multi-ethnic cohort. We also examined their relationship in pre-specified subgroups by age, sex, ethnicity and their baseline tolerance to a glucose load using standard oral glucose tolerance test.

\section{METHODS}

\section{Study settings and population}

The Insulin Resistance Atherosclerosis Study (IRAS) is a multi-ethnic, multi-center observational cohort study. The study design and selection criteria for the IRAS cohort have been previously described. ${ }^{9}$ Participants were recruited at four clinical centers between October 1992 and April 1994. Ethnicity was determined by self-report. Two of the clinical centers (Los Angeles, CA, USA and Oakland, CA, USA) were assigned to recruit African-American and non-Hispanic white participants aged between 40 and 69 years. In these centers, individuals were sampled from the members of a non-profit health maintenance organization. In the other two clinical centers (San Luis Valley, CA, USA and San Antonio, TX, USA), Hispanic and non-Hispanic white participants were recruited from ongoing population-based epidemiologic studies. We excluded participants with coronary heart disease and cancer. All participants provided informed consent as approved by their respective field center's institutional review board. For the purpose of the current evaluation of the association of fatty acids with incident type 2 diabetes, a subcohort of individuals without baseline diabetes was chosen $(n=749)$. Participants with missing fatty acids $(\mathrm{n}=30)$ were excluded.

\section{Covariate assessment}

Clinical and anthropometric measurements, medical history and demographic information were obtained at baseline from all the participants. Sex, ethnicity, history of type 2 diabetes in parents, alcohol use, educational status and smoking habits were obtained by self-report. Education was dichotomized into <high school and high school, some college or vocational school, college graduate. All participants had a frequently sampled intravenous glucose tolerance test with Minimal-model analysis (MINMOD) analysis for measurement of insulin sensitivity $\left(\mathrm{S}_{\mathrm{I}}\right)$ and AIR. ${ }^{10}$ AIR is a measure of pancreatic function and insulin release. Oral glucose tolerance tests were administered to determine glucose tolerance status (normal, impaired glucose tolerance or type 2 diabetes), based on the 2018 American Diabetes Association criteria for fasting or 2-hour post-load glucose concentrations, and oral hypoglycemic agent or insulin use. ${ }^{11}$ Height and weight were obtained and body mass index (BMI) was calculated. Total cholesterol, low-density lipoprotein and high-density lipoproteins were measured at a central laboratory. ${ }^{9}$ Physical activity was assessed using a validated physical activity recall which calculated total estimated energy expenditure (kcal/ $\mathrm{kg}$ /year) over the past year as the sum of energy expenditure from activities and sleep. Usual dietary intake over the previous year prior to baseline examination was assessed using a 114-item food frequency questionnaire (FFQ) modified from the National Cancer Institute's Health Habits and History Questionnaire to include ethnic and regional foods relevant to the study population. These methods have been described previously. ${ }^{12}$ The validity and reproducibility of this FFQ were established in a subsample of 186 women from the IRAS population using eight 24 hours dietary recalls, followed by a second FFQ. The daily carbohydrate intake was calculated in grams.

\section{Fatty acid measurement}

We measured fatty acids in the fasting plasma samples, utilizing a targeted, quantitative gas chromatography approach which has been previously described (Lipomics Technologies). ${ }^{13}$ Briefly, the lipids from plasma were extracted in the presence of authentic internal standards using chloroform:methanol $(2: 1 \mathrm{v} / \mathrm{v})$, the Folch method. ${ }^{14}$ The total lipid extract was trans-esterified in $1 \%$ sulfuric acid in methanol in a sealed vial under a nitrogen atmosphere at $100^{\circ} \mathrm{C}$ for $45 \mathrm{~min}$. The resulting samples were neutralized with $6 \%$ potassium carbonate and the fatty acid methyl esters (FAMEs) re-extracted with hexane and prepared for gas chromatography. FAMEs were separated and quantified by capillary gas chromatography (Agilent Technologies model 6890) equipped with a $30 \mathrm{~m}$ DB-88MS capillary column (Agilent Technologies) and a flame-ionization detector. Quantitative results were obtained by comparing each fatty acid to its internal standard control. All determinations presented here passed internal quality assurance and quality control processes.

This study focused entirely on the relationship between fatty acid metabolism and type 2 diabetes, not on the relationship between total plasma lipid levels and type 2 diabetes. Thus, we chose to use mole percent data ( $\mathrm{mol} \%)$ for all analyses. Mol\% data are simply fatty acid composition data, with each fatty acid expressed as a percentage of molar mass of the total pool of triglyceride fatty acid molar mass. By converting quantitative fatty acid concentrations to $\mathrm{mol} \%$, the effect of changes in total plasma lipid levels (eg, increased or decreased triglyceride) was normalized. Test-retest reliability coefficients were $>0.8$ for all plasma fatty acids. 


\section{Outcome}

In IRAS, participants were followed up in 1997-1999 after a period of 5 years from study baseline (1992-1994). Fasting and 2-hour post-challenge glucose concentration, glycated hemoglobin and use of insulin or oral hypoglycemic medication was ascertained at baseline by trained technicians. Type 2 diabetes was defined as (1) fasting glucose concentration $\geq 126 \mathrm{mg} / \mathrm{dL} \quad(7 \mathrm{mmol} / \mathrm{L})$, (2) 2-hour post-oral glucose tolerance test glucose concentration $\geq 200 \mathrm{mg} / \mathrm{dL}(11 \mathrm{mmol} / \mathrm{L})$, (3) new use of an insulin or oral hypoglycemic medication, (4) fasting or non-fasting haemoglobin A1c (HbAlc) concentration $\geq 6.5 \%$, or (5) self-identified type 2 diabetes. ${ }^{11}$

\section{Statistical analysis}

Descriptive analyses including $\chi^{2}$ tests, t-tests and analysis of variance were performed after examination of data for outliers and application of variance stabilizing transformations, when appropriate. Plasma fatty acids were analyzed in SD units in multivariable regression models. The associations of plasma fatty acids (mol\%) and their ratios with incident type 2 diabetes were examined in multivariable logistic regression models adjusted for covariates. Model 1 included age, sex, ethnicity, BMI, carbohydrate intake, oral glucose tolerance status, physical activity, family history of diabetes, alcohol, smoking status and education. Carbohydrate intake was included in the model based on studies demonstrating its role in stimulating DNL. ${ }^{15}$ Model 2 included model 1 covariates in addition to insulin sensitivity, while model 3 included model 2 covariates and AIR. The ratios of 16:1 n-7/16:0 and 18:1 n-9/18:0 reflect activity of (microsomal) stearoyl CoA desaturase-1 (SCD1) $\Delta$ 9-desaturase while the ratio of 16:0/18:0 reflected the activity of elongation of longchain fatty acids family member 6 (Elovl6) enzyme. ${ }^{16}$ We also examined associations of fatty acids with incident type 2 diabetes within pre-specified subgroups of median age (55 years), sex, ethnicity and normal versus impaired glucose tolerance. We tested for interactions between fatty acids and age, sex, glucose tolerance status in logistic regression models adjusted for variables in model 3 . We also constructed a restricted cubic spline model to examine the linearity of the relationship between fatty acid plasma concentrations and the risk of type 2 diabetes, with adjustment for variables in model 3, incorporating knots at the 5th, 50th and 95th percentiles. ${ }^{18}$ All analyses were performed using PASW V.18.0 (IBM, Chicago, IL, USA) and SAS V.9.3 (SAS institute Cary, NC, USA).

\section{RESULTS}

A total of 719 participants (mean age $55.1 \pm 8.5$ years, 44.2\% men, $42.3 \%$ Caucasians, $24.5 \%$ African-Americans, $33.2 \%$ Hispanics) had data available for fatty acids. Baseline characteristics stratified by incident type 2 diabetes status are given in table 1 . There were
$146(20.3 \%)$ participants that developed diabetes at the end of 5-year follow-up. Participants with incident type 2 diabetes had higher fasting blood glucose and lower insulin sensitivity and lower AIR. Incident type 2 diabetes cases also had higher baseline BMI, compared with those who did not convert to type 2 diabetes at follow-up, in line with the extensive literature showing this association. ${ }^{19}$ There were also significant differences among fatty acids at baseline. Participants that developed incident type 2 diabetes had significantly higher levels of palmitic, palmitoleic and oleic acids.

Table 2 shows the correlations between the fatty acids that were measured in the current study. Stearic acid was negatively correlated with myristic and palmitic acid while palmitic acid was highly correlated with palmitoleic acid.

Table 3 shows the risk of type 2 diabetes associated with fatty acids and their ratios. Note, the associations of the fatty acids with incident type 2 diabetes did not differ across ethnicities. Palmitic acid had the strongest association after adjusting for demographics, BMI, carbohydrate intake, physical activity, family history of diabetes, alcohol, smoking status and education (OR per SD unit $1.44 ; 95 \%$ CI 1.18 to $1.74, \mathrm{p}<0.001)$ which was not attenuated with the inclusion of insulin sensitivity and AIR in the multivariable adjusted model (OR 1.36; 95\% CI 1.09 to $1.70, \mathrm{p}=0.01)$. Similarly, palmitoleic and oleic acids were also significantly and independently associated with incident type 2 diabetes.

There was also an inverse association of stearic acid to palmitic acid ratio with type 2 diabetes, which signifies that increased expression of Elovl6 enzyme was associated with decreased 5-year risk of type 2 diabetes. On the other hand, the ratio of palmitoleic acid to palmitic acid and oleic acid to stearic acid, which signifies an increased expression of SCD1 (involved in both pathways), was associated with increased 5-year risk of incident type 2 diabetes (table 3 ). These ratios were slightly attenuated, but remained despite additional adjustment for insulin sensitivity and AIR. We also conducted a sensitivity analysis adjusting for the 5-year change in $\mathrm{BMI}$, rather than baseline BMI and found no changes in the direction and magnitude of the associations overall, although the associations with oleic acid and oleic acid/stearic acid (SCD1) were attenuated (online supplementary table 1 ).

In addition, we analyzed associations of fatty acids that were significantly associated with type 2 diabetes in the analysis described above among pre-specified subgroups (median age, sex and glucose tolerance status) (figure 1). There were no significant interactions of age, sex or glucose tolerance status noted for associations of fatty acids with incident type 2 diabetes. The associations of fatty acids with risk of incident type 2 diabetes adjusted for model 3 variables across increasing fatty acid mol\% are shown in supplemental figure $\mathrm{S} 1$. The three knots were placed at 5 th, median and 95th percentiles. The ORs for risk of incident type 
Table 1 Baseline characteristics of the cohort stratified by diabetes status at 5-year follow-up

\begin{tabular}{|c|c|c|c|c|}
\hline Characteristic & Overall & Yes & No & $P$ value \\
\hline $\mathrm{N}$ & 719 & $146(20.3 \%)$ & $573(79.7 \%)$ & \\
\hline Age (years) & $55.1 \pm 8.5$ & $55.7 \pm 8.1$ & $54.9 \pm 8.6$ & 0.33 \\
\hline Males & $318(44.2 \%)$ & $57(39.0 \%)$ & $261(45.5 \%)$ & 0.16 \\
\hline \multicolumn{5}{|l|}{ Race/ethnicity } \\
\hline Whites & $304(42.3 \%)$ & $58(39.7 \%)$ & $246(42.9 \%)$ & 0.48 \\
\hline Hispanics & $239(33.2 \%)$ & $52(35.6 \%)$ & $187(32.6 \%)$ & 0.49 \\
\hline African-Americans & $176(24.5 \%)$ & $36(24.7 \%)$ & $140(24.4 \%)$ & $>0.99$ \\
\hline Body mass index $\left(\mathrm{kg} / \mathrm{m}^{2}\right)$ & $28.4 \pm 5.6$ & $31.4 \pm 6.7$ & $27.7 \pm 5.0$ & $<0.001$ \\
\hline Fasting glucose (mg/dL) & $98.1 \pm 11.1$ & $106.5 \pm 12.6$ & $96.0 \pm 9.7$ & $<0.001$ \\
\hline Acute insulin response $(\mu \mathrm{U} / \mathrm{ml})$ & $52.6 \pm 49.7$ & $31.6 \pm 32.8$ & $57.8 \pm 51.9$ & $<0.001$ \\
\hline Insulin sensitivity $\left(\times 10^{-4} \mathrm{~min}^{-1}\left[\mu \mathrm{U}^{\prime} \mathrm{ml}\right]^{-1}\right)$ & $2.2 \pm 2.1$ & $1.3 \pm 1.6$ & $2.4 \pm 2.2$ & $<0.001$ \\
\hline Family history of diabetes & $285(39.6 \%)$ & $73(50.0 \%)$ & $212(37.0 \%)$ & 0.01 \\
\hline Education $>12$ grade & $138(19.2 \%)$ & $24(16.4 \%)$ & $114(19.9 \%)$ & 0.20 \\
\hline Impaired glucose tolerance & $244(33.9 \%)$ & $94(64.4 \%)$ & $150(26.2 \%)$ & $<0.001$ \\
\hline Estimated energy expenditure (kcal/kg/year) & $14855.9 \pm 2739.3$ & $14819.6 \pm 2578.1$ & $14865.1 \pm 2780.7$ & 0.86 \\
\hline Alcohol use & & & & 0.15 \\
\hline Current & $370(51.5 \%)$ & $80(54.8 \%)$ & $290(50.6 \%)$ & \\
\hline Former & $267(37.1 \%)$ & $45(30.8 \%)$ & $222(38.7 \%)$ & \\
\hline Smoking history & & & & 0.01 \\
\hline Current & $105(14.6 \%)$ & $33(22.6 \%)$ & $72(12.6 \%)$ & \\
\hline Former & $282(39.2 \%)$ & 49 (33.6\%) & $233(40.7 \%)$ & \\
\hline Dietary carbohydrate intake (g/day) & $214.9 \pm 86.5$ & $209.8 \pm 89.5$ & $216.2 \pm 85.7$ & 0.42 \\
\hline Myristic acid (14:0) & $1.0 \pm 0.4$ & $1.1 \pm 0.3$ & $1.0 \pm 0.4$ & 0.24 \\
\hline Palmitic acid (16:0) & $21.6 \pm 2.0$ & $22.3 \pm 2.1$ & $21.5 \pm 2.0$ & $<0.001$ \\
\hline Stearic acid (18:0) & $6.8 \pm 0.7$ & $6.8 \pm 0.7$ & $6.8 \pm 0.7$ & $>0.99$ \\
\hline Myristoleic acid (14:1n5) & $0.1 \pm 0.05$ & $0.1 \pm 0.05$ & $0.1 \pm 0.05$ & 0.88 \\
\hline Palmitoleic acid (16:1n7) & $2.2 \pm 0.9$ & $2.5 \pm 1.0$ & $2.2 \pm 0.9$ & $<0.001$ \\
\hline Oleic acid (18:1n9) & $19.6 \pm 2.7$ & $20.2 \pm 2.6$ & $19.4 \pm 0.2 .7$ & 0.001 \\
\hline
\end{tabular}

Mean and SD, or $\mathrm{N}$ and percent. Fatty acids are mol\%.

2 diabetes are demonstrated in reference to the median plasma levels of fatty acids.

\section{CONCLUSIONS}

In this prospective multi-ethnic cohort, we investigated the associations of metabolic perturbations in fatty acid metabolism with 5-year risk of incident type 2 diabetes, before and after adjusting for measures of insulin sensitivity and AIR. We identified that there were significant associations of these plasma fatty acids with incident type 2 diabetes which remained after adjusting for insulin sensitivity or AIR. We found that the strongest association with

Table 2 Correlations between the fatty acids

\begin{tabular}{|c|c|c|c|c|c|c|}
\hline Fatty acids & $\begin{array}{l}\text { Myristic } \\
\text { acid (14:0) }\end{array}$ & $\begin{array}{l}\text { Palmitic } \\
\text { acid (16:0) }\end{array}$ & $\begin{array}{l}\text { Stearic acid } \\
(18: 0)\end{array}$ & $\begin{array}{l}\text { Myristoleic acid } \\
(14: 1 n 5)\end{array}$ & $\begin{array}{l}\text { Palmitoleic acid } \\
(16: 1 \mathrm{n} 7)\end{array}$ & $\begin{array}{l}\text { Oleic acid } \\
(18: 1 \mathrm{n} 9)\end{array}$ \\
\hline Myristic acid (14:0) & 1 & $0.623^{* *}$ & $-0.083^{* *}$ & $0.848^{* *}$ & $0.502^{* *}$ & $0.360^{* *}$ \\
\hline Palmitic acid (16:0) & & 1 & $-0.278^{*}$ & $0.503^{* *}$ & $0.725^{* *}$ & $0.422^{* *}$ \\
\hline Stearic acid (18:0) & & & 1 & 0.008 & $-0.333^{* *}$ & $-0.312^{*}$ \\
\hline Myristoleic acid (14:1n5) & & & & 1 & $0.550^{* *}$ & $0.334^{* *}$ \\
\hline Palmitoleic acid (16:1n7) & & & & & 1 & $0.502^{* *}$ \\
\hline Oleic acid (18:1n9) & & & & & & 1 \\
\hline
\end{tabular}

${ }^{*} p<0.05$

${ }^{* \star} p<0.01$. 


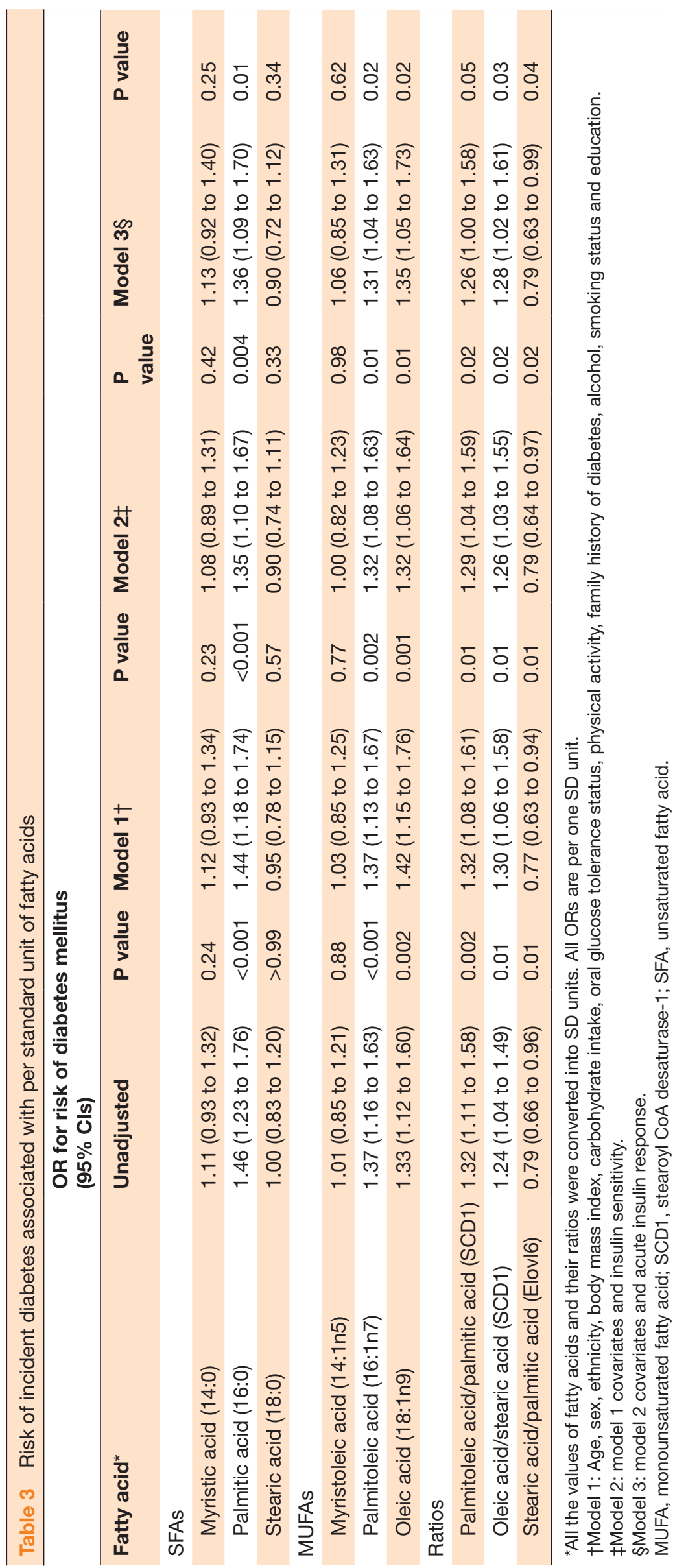




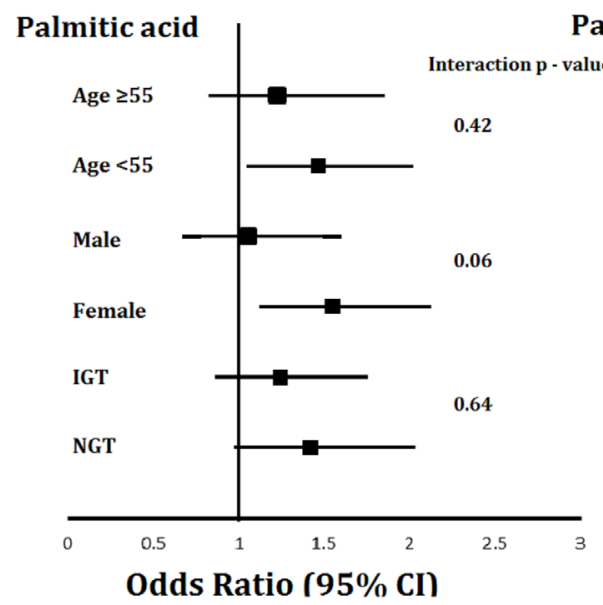

Palmitoleic acid

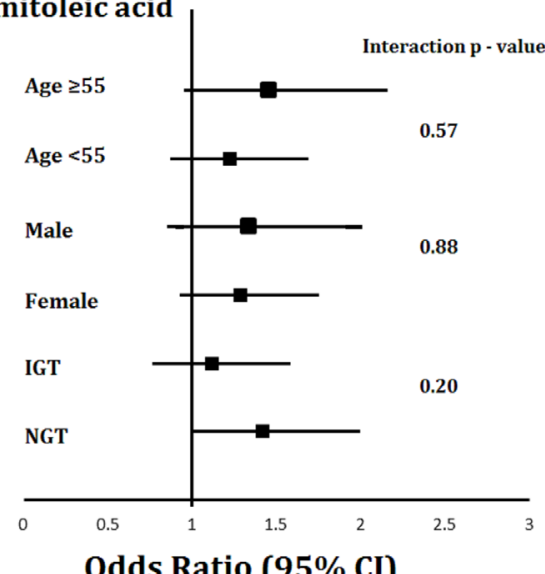

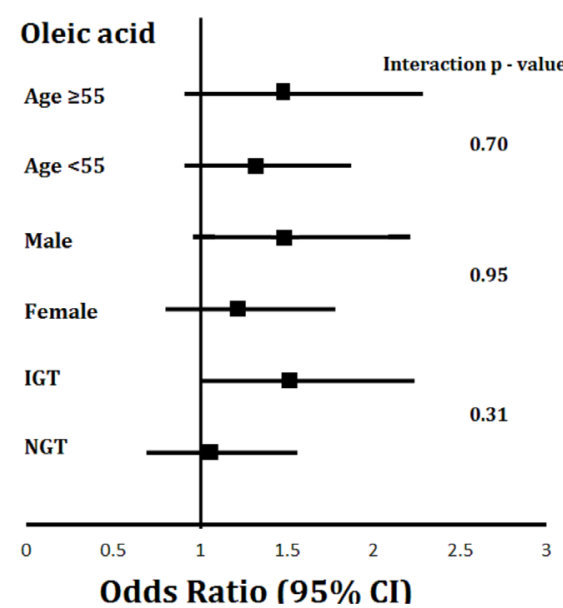

Figure 1 Subgroup analyses of association of palmitic acid, oleic acid and palmitoleic acids.

risk of type 2 diabetes was with palmitic acid (adjusted OR $1.36 ; 95 \%$ CI 1.09 to $1.70, p=0.01$ ). The ratio of stearic acid to palmitic acid was inversely associated with risk of type 2 diabetes, while ratio of palmitoleic acid to palmitic acid and oleic acid to stearic acid (SCD1 activity) was directly associated with risk of incident type 2 diabetes. There were no significant interactions for ethnicity, age, sex or oral glucose tolerance status with the main effects.

A limited number of previous studies have examined the association of individual circulating DNL fatty acids with type 2 diabetes incidence. The European Prospective Investigation into Cancer and Nutrition (EPIC)-InterAct case-cohort study, which included 12403 incident type 2 diabetes cases and 16154 controls, found that plasma even-chain saturated fatty acids, myristic, palmitoleic and stearic acid, were positively associated with incident type 2 diabetes. Similarly, SCD-1 marker, palmitoleic acid to palmitic acid ratio, but not oleic acid to stearic acid ratio, was positively associated with incident type 2 diabetes, ${ }^{20}$ a finding which has also been reported in other smaller observational studies. ${ }^{37}$ In 2274 participants of the EPICPotsdam study, only palmitoleic acid, stearic acid and oleic acids in erythrocyte membranes were directly associated with risk of incident type 2 diabetes while palmitic and myristic acids were not associated. ${ }^{7}$ The study concluded that fatty acids that reflect SCD1 activity are associated with risk of incident type 2 diabetes. In another nested case-control study of 159 diabetic cases and 291 control individuals, erythrocyte membrane fatty acids, myristic, palmitic, palmitoleic acid differed significantly between the cases and controls, with higher levels in those with diabetes. However, oleic acid did not differ significantly between the groups. The authors of this study found significant direct associations of myristic, palmitic, palmitoleic, stearic acid and SCD-1 (palmitoleic acid to palmitic acid ratio) but not SCD-1 (oleic acid to stearic acid ratio) with incident DM. However, these associations did not remain significant after adjustment of HbAlc and BMI in the models. ${ }^{3}$ In a prospective evaluation of 3004 Cardiovascular Health Study participants (mostly Caucasians), plasma phospholipid palmitic acid and stearic acid, but not oleic acid, was associated with higher type 2 diabetes risk. ${ }^{21}$ Furthermore, in a cross-sectional study using National Health and Nutrition Examination Survey (NHANES) data (2003-2004), elevated plasma myristic, palmitic, stearic and oleic acid concentrations were associated with elevated fasting plasma glucose and HbAlc. ${ }^{4}$ The findings of our study are consistent with the majority of these previous findings by showing a direct relationship of myristic, palmitic, palmitoleic, myristoleic and oleic acid with incident DM. We, however, did not find association of stearic acid in unadjusted or adjusted models. Taken together, studies examining individual circulating DNL fatty acids on type 2 diabetes incidence are still inconsistent. Further research into the varying effects of individual fatty acids on type 2 diabetes risk and the possible mechanisms of action of DNL fatty acids on type 2 diabetes pathophysiology are needed. Heterogeneity in the current literature may partly explained by differences in fatty acid measurement (total plasma vs erythrocyte membrane), variations in interindividual lipid metabolism and population characteristics.

Our results provide a novel contribution to the existing literature on the role of DNL fatty acids on risk of type 2 diabetes. Specifically, we demonstrated that adjustment of our models for directly measured insulin sensitivity and insulin secretion did not substantially alter the associations of fatty acids involved in DNL with type 2 diabetes risk. This is a notable observation, as insulin sensitivity and secretion represent the major upstream pathophysiological disorders underlying type 2 diabetes risk. Past studies did not include insulin sensitivity or insulin release parameters. Furthermore, we also observed both the ratios of palmitoleic acid to palmitic acid (SCD1) and the ratio of oleic acid to stearic acid (SCD1) were associated with diabetes. Both of the products are governed by a rate limiting desaturase enzyme, $\mathrm{SCD} 1$. The conversion step of palmitic acid to palmitoleic acid that is dependent on SCD1 activity mainly occurs in hepatocytes and thus has been previously shown to play a major role in body weight, hepatic steatosis and obesity regulation. ${ }^{22}{ }^{23}$ Our study adds to this literature by demonstrating a potential 
role of SCD1 in development of type 2 diabetes, independent of these factors. This also suggests that these other phenotypes could also contribute to type 2 diabetes pathophysiology through insulin resistance independent pathways. Inhibitors of SCD1 are under investigation for type 2 diabetes treatment, which might involve anti-sense oligonucleotide or RNA interference. ${ }^{24}{ }^{25}$ Mechanistic studies examining DNL-derived fatty acids and associated enzymes as biomarkers or predictors of type 2 diabetes development are warranted. Furthermore, additional studies evaluating factors, including metabolic, dietary and gene-environment interactions, that may influence levels of DNL fatty acids in circulation need to be examined. Previous animal and in vitro studies have demonstrated lipotoxic properties of even-chained saturated fatty acids, with high levels of these fatty acids in circulation resulting in insulin resistance, inflammation and endoplasmic reticulum stress. In addition, previous IRAS studies have demonstrated that diets high in saturated fatty acids, as measured through a lower adherence to a DASH-type diet (Dietary Approaches to Stop Hypertension) ${ }^{26}$ lower circulating plasma fatty acids derived from dairy consumption ${ }^{27}$ and lower intake of a 'healthy" diet pattern identified through reduced rank regression, were associated with incident type 2 diabetes. ${ }^{28} \mathrm{High}$ DNL rates have also been shown to contribute to insulin resistance and hepatic steatosis. ${ }^{21}$

There are several limitations of the study, which include a relatively modest sample size and the lack of a validation cohort. In addition, the exact time of development of type 2 diabetes was not available since diabetes status was determined at the 5-year follow-up clinic examination using oral glucose tolerance tests. However, as a progressive disease, the actual time of diagnosis of type 2 diabetes is difficult to determine precisely. Fatty acids were only measured as baseline; therefore, any temporal changes in exposure-outcome associations cannot be determined. Moreover, this was a cohort study and thus causality cannot be established. Furthermore, individuals were enrolled on volunteer basis thus healthy cohort effect (individuals might have healthier behaviors than rest of the community) might be present; this phenomenon is difficult to avoid in epidemiologic studies. Finally, there may be residual confounding that may explain the associations observed.

In conclusion, this study demonstrated the relationship of fatty acids involved in the DNL pathway with 5 -year risk of type 2 diabetes, independent of detailed measures of insulin resistance and insulin release. The relative ratios of fatty acids and risk of type 2 diabetes suggest an important role of SCD1 in development of type 2 diabetes, which was uniform across all strata of age, sex, ethnicity and glucose tolerance. A better understanding of this pathway and its inhibitors may lead to development of effective type 2 diabetes preventive strategies.
Author affiliations

${ }^{1}$ Department of Internal Medicine, Wake Forest University, Winston Salem, North Carolina, USA

${ }^{2}$ Department of Nutritional Sciences, University of Toronto, Toronto, Ontario, Canada ${ }^{3}$ Department of Lipomics, Metabolon Inc, West Sacramento, North Carolina, USA ${ }^{4}$ Department of Medicine, University of Texas Health Science Center at San Antonio, San Antonio, Texas, USA

${ }^{5}$ Department of Public Health Sciences, Wake Forest University, Winston-Salem, North Carolina, USA

Contributors WQ wrote the manuscript and researched data. IDS wrote/reviewed/ edited the manuscript and revisions. AH reviewed/edited manuscript. SKW researched data and reviewed/edited manuscript. CL reviewed/edited manuscript. LEW reviewed/edited and wrote the manuscript. AH is the guarantor of this work and, as such, had full access to all of the data in the study and takes responsibility for the integrity of the data and the accuracy of the data analysis.

Funding This work was supported by grants U01-HL-47892, U01-HL-47902, DK-29867, R01-58329 and DK-079888 from the National Heart, Lung, and Blood Institute and grant M01-RR-43 from the National Institutes of Health. AJH holds a Tier II Canada Research Chair in the Epidemiology of Type 2 Diabetes. Waqas Qureshi reports receiving Ruth L Kirschstein NRSA Institutional Training Grant 5T32HL076132-10.

Competing interests None declared.

Patient consent for publication Not required.

Provenance and peer review Not commissioned; externally peer reviewed.

Data availability statement No data are available.

Open access This is an open access article distributed in accordance with the Creative Commons Attribution Non Commercial (CC BY-NC 4.0) license, which permits others to distribute, remix, adapt, build upon this work non-commercially, and license their derivative works on different terms, provided the original work is properly cited, appropriate credit is given, any changes made indicated, and the use is non-commercial. See: http://creativecommons.org/licenses/by-nc/4.0/.

\section{REFERENCES}

1. Cho NH, Shaw JE, Karuranga S, et al. IDF diabetes atlas: global estimates of diabetes prevalence for 2017 and projections for 2045. Diabetes Res Clin Pract 2018;138:271-81.

2. American Diabetes Association. Economic costs of diabetes in the U.S. in 2017. Diabetes Care 2018;41:917-28.

3. Krachler B, Norberg M, Eriksson JW, et al. Fatty acid profile of the erythrocyte membrane preceding development of type 2 diabetes mellitus. Nutr Metab Cardiovasc Dis 2008;18:503-10.

4. Yu EA, Hu PJ, Mehta S. Plasma fatty acids in de novo lipogenesis pathway are associated with diabetogenic indicators among adults: NHANES 2003-2004. Am J Clin Nutr 2018;108:622-32.

5. Hodge AM, English DR, O'Dea K, et al. Plasma phospholipid and dietary fatty acids as predictors of type 2 diabetes: interpreting the role of linoleic acid. Am J Clin Nutr 2007;86:189-97.

6. Wang L, Folsom AR, Zheng Z-J, et al. Plasma fatty acid composition and incidence of diabetes in middle-aged adults: the Atherosclerosis risk in communities (ARIC) study. Am J Clin Nutr 2003;78:91-8.

7. Kröger J, Zietemann V, Enzenbach C, et al. Erythrocyte membrane phospholipid fatty acids, desaturase activity, and dietary fatty acids in relation to risk of type 2 diabetes in the European Prospective Investigation into Cancer and Nutrition (EPIC)-Potsdam Study. Am J Clin Nutr 2011;93:127-42.

8. Patel PS, Sharp SJ, Jansen E, et al. Fatty acids measured in plasma and erythrocyte-membrane phospholipids and derived by food-frequency questionnaire and the risk of new-onset type 2 diabetes: a pilot study in the European Prospective Investigation into Cancer and Nutrition (EPIC)-Norfolk cohort. Am J Clin Nutr 2010;92:1214-22.

9. Wagenknecht LE, Mayer EJ, Rewers M, et al. The insulin resistance atherosclerosis study (IRAS) objectives, design, and recruitment results. Ann Epidemiol 1995;5:464-72.

10. Pacini G, Bergman RN. MINMOD: a computer program to calculate insulin sensitivity and pancreatic responsivity from the frequently sampled intravenous glucose tolerance test. Comput Methods Programs Biomed 1986;23:113-22.

11. American Diabetes Association. 2. Classification and Diagnosis of Diabetes: Standards of Medical Care in Diabetes-2018. Diabetes Care 2018;41(Suppl 1):S13-S27. 
12. Mayer-Davis EJ, Vitolins MZ, Carmichael SL, et al. Validity and reproducibility of a food frequency interview in a Multi-Cultural epidemiology study. Ann Epidemiol 1999;9:314-24.

13. Watkins SM, Reifsnyder PR, Pan $\mathrm{H}$-ju, et al. Lipid metabolomewide effects of the PPARgamma agonist rosiglitazone. $J$ Lipid Res 2002;43:1809-17.

14. Folch J, Lees M, SLOANE STANLEY GH. A simple method for the isolation and purification of total lipides from animal tissues. $J$ Biol Chem 1957;226:497-509.

15. Hodson L, Skeaff CM, Fielding BA. Fatty acid composition of adipose tissue and blood in humans and its use as a biomarker of dietary intake. Prog Lipid Res 2008;47:348-80.

16. Jakobsson A, Westerberg R, Jacobsson A. Fatty acid elongases in mammals: their regulation and roles in metabolism. Prog Lipid Res 2006:45:237-49.

17. Ntambi JM, Miyazaki M. Regulation of stearoyl-CoA desaturases and role in metabolism. Prog Lipid Res 2004;43:91-104.

18. Marrie RA, Dawson NV, Garland A. Quantile regression and restricted cubic splines are useful for exploring relationships between continuous variables. J Clin Epidemiol 2009;62:511-7.

19. Ganz ML, Wintfeld N, Li Q, et al. The association of body mass index with the risk of type 2 diabetes: a case-control study nested in an electronic health records system in the United States. Diabetol Metab Syndr 2014;6.

20. Forouhi NG, Koulman A, Sharp SJ, et al. Differences in the prospective association between individual plasma phospholipid saturated fatty acids and incident type 2 diabetes: the EPIC-InterAct case-cohort study. Lancet Diabetes Endocrinol 2014;2:810-8.
21. Ma W, Wu JHY, Wang Q, et al. Prospective association of fatty acids in the de novo lipogenesis pathway with risk of type 2 diabetes: the cardiovascular health study. Am J Clin Nutr 2015;101:153-63.

22. Miyazaki T, Shimada K, Hiki M, et al. High hexacosanoic acid levels are associated with coronary artery disease. Atherosclerosis 2014;233:429-33.

23. Dobrzyn A, Dobrzyn P, Lee S-H, et al. Stearoyl-Coa desaturase-1 deficiency reduces ceramide synthesis by downregulating serine palmitoyltransferase and increasing beta-oxidation in skeletal muscle. Am J Physiol Endocrinol Metab 2005;288:E599-E607.

24. Liu G. Recent advances in stearoyl-CoA desaturase 1 inhibitors for dyslipidemia and obesity. Curr Top Med Chem 2010;10:419-33.

25. Iida T, Ubukata M, Mitani I, et al. Discovery of potent liver-selective stearoyl-CoA desaturase-1 (SCD1) inhibitors, thiazole-4-acetic acid derivatives, for the treatment of diabetes, hepatic steatosis, and obesity. Eur J Med Chem 2018;158:832-52.

26. Liese AD, Nichols M, Sun X, et al. Adherence to the DASH diet is inversely associated with incidence of type 2 diabetes: the insulin resistance atherosclerosis study. Diabetes Care 2009;32:1434-6.

27. Santaren ID, Watkins SM, Liese AD, et al. Serum pentadecanoic acid (15:0), a short-term marker of dairy food intake, is inversely associated with incident type 2 diabetes and its underlying disorders. Am J Clin Nutr 2014;100:1532-40.

28. Liese $A D$, Weis KE, Schulz M, et al. Food intake patterns associated with incident type 2 diabetes: the insulin resistance atherosclerosis study. Diabetes Care 2009;32:263-8. 